\title{
Desenvolvimento de uma Escala de Integração ao Ensino Superior ${ }^{1}$
}

\author{
Soely A. J. Polydoro ${ }^{2}$ \\ Ricardo Primi \\ Maria de Nazaré da F. Serpa \\ Margarida M. Hoeppner Zaroni \\ Kelly Cristina Pereira Pombal
}

\begin{abstract}
Resumo
Este trabalho apresenta o desenvolvimento de uma Escala de Integração ao Ensino Superior (EIES). A amostra foi constituída por 633 universitários de séries iniciais e finais de sete cursos das áreas Humanas, Exatas e Biológicas. O instrumento foi composto por 46 itens em forma de escala. A consistência interna da escala geral foi igual a 0,86 . A análise fatorial exploratória agrupou os itens em 12 subescalas. Por meio da análise fatorial confirmatória corroborou-se a organização das subescalas em dois fatores, um associado aos aspectos externos referentes ao ambiente universitário, de satisfação e aderência ao curso, e outro, aos aspectos internos de capacidade de enfrentamento, reações físicas psicossomáticas e estado de humor do estudante.

Palavras-chave: Caracterização de universitários; ambiente universitário; avaliação psicológica; medidas de satisfação.
\end{abstract}

\section{Development of a Scale for Student Adaptation to Academic Enviroment Assessment}

\begin{abstract}
This research presents the development of a scale to assess student's adaptation to academic environment. A sample of 633 students from humanities, engineering and biological colleges answered to the questionnaire. The scale included 46 items and the full-scale alpha reliability coefficient was .86. The exploratory factor analysis resulted in 12 -factor solution. Using confirmatory factor analysis it was corroborated a two broader factor organization one referring to external aspects including college environment, satisfaction, and attachment, the other referring to internal aspects including coping, physical reactions, and humor.

Keywords: Academic environment; college student characteristics; psychological assessment; and measures of satisfaction.
\end{abstract}

\section{Introdução}

Este estudo procurou desenvolver um instrumento para avaliação da integração do estudante ao ensino superior para ser utilizado em uma pesquisa mais ampla de caracterização psicológica do universitário quanto a personalidade, estilos de pensamento e integração acadêmica à universidade e sua relação com área de conhecimento e fase de formação.

Muitas pesquisas têm tido o universitário como sujeito, especialmente no que se refere à investigação do perfil social e econômico (Bezzon, 1995; Primi, Vendramini, Santos, \& Figueiredo, 1999), condições de estudo (Carelli, 1996; Mercuri, 1992), aproveitamento (Larose \& Roy, 1991; Pacheco, 1996), habilidades básicas e aprendizagem (Santos e cols. 2000) Outras variáveis também têm sido estudadas, como a compreensão da realidade vivencial dos alunos (Calejon, 1996). No entanto, esses elementos não são isolados.

A integração à universidade é um processo multifacetado construído no cotidiano das relações que se estabelecem entre o estudante e a instituição. Caracteriza-

\footnotetext{
1 Esse trabalho faz parte de um projeto de pesquisa financiado pela Universidade São Francisco (USF) pelo Programa PEPCI/USF.

${ }^{1}$ Endereço para correspondência:

Centro de Ciências Humanas e Sociais, Rua Alexandre Rodrigues Barbosa, 45, Itatiba-SP; 13251-900.
}

se pela troca entre as expectativas, características e habilidades dos estudantes e a estrutura, normas e a comunidade que compõem a universidade.

A integração à Universidade tem sido a variável central na decisão entre a permanência ou abandono do objetivo de graduação (Bean, 1980; Brower, 1992; Cabrera, Castañeda, Nora et al.,1992; Cabrera, Nora \& Castañeda, 1993; Gerder \& Mallinckrodt, 1994; Tinto, 1975). A literatura sobre a evasão no ensino superior afirma que a desistência é mais provável para o estudante que estiver insuficientemente integrado acadêmica e socialmente à universidade (Christie \& Dinham, 1991; Terenzini, Redon, Upcraft et al., 1994; Tinto, 1975). Inclusive, como a revisão de Baker e Siryk (1989) demonstra, grande parte dos instrumentos anteriormente criados para a avaliação da integração acadêmica tinham como preocupação básica prever a evasão universitária a partir da medida de integração (Caio, 1996).

Baker e Siryk (1989) identificaram quatro dimensões relacionadas à integração do estudante à universidade: (a) o ajustamento acadêmico: referindo-se ao atendimento das demandas educacionais que a instituição apresenta ao estudante; (b) o ajustamento relacionalsocial: referindo-se às demandas interpessoais e sociais da vida universitária; (c) o ajustamento pessoal-emocional: referindo-se ao estado psicológico e físico do uni- 
versitário; e (d) o comprometimento com a instituição/aderência: referindo-se à qualidade da ligação entre o estudante e o curso e do estudante com a instituição.

Como referem esses autores, o ajustamento do estudante à universidade envolve diferentes demandas de respostas de enfrentamento ou ajustamento que por sua vez, podem possuir eficácia variada.

A aprendizagem das respostas que esse novo ambiente exige deve ser ágil, indo desde o domínio do vocabulário acadêmico e do espaço físico, até a assimilação dos valores e procedimentos acadêmicos (Almeida, 1998; Arruda, Millan, Rossi e col.,1994; Frost,1993; Millan e Barbedo, 1994).

A possibilidade de adaptação à transição é peculiar a cada um, sendo determinada pela interação de dois conjuntos de fatores: (a) fatores internos como por exemplo, a vulnerabilidade do indivíduo ao estresse; e (b) fatores externos como a complexidade e capacidade do ambiente em responder às necessidades do indivíduo e às características do próprio estresse - fonte, duração e grau (Felner e Adan, 1990; Gunter e Gunter, 1986).

Assim, apesar de presentes no mesmo ambiente universitário, a história de cada estudante irá definir diferentes formas de interação com a universidade (Mercuri, Moran e Azzi, 1995). Para Tinto (1975) esse processo de interações entre o universitário e os sistemas acadêmicos e sociais da instituição ocorre ao longo da graduação, modificando continuamente o compromisso do estudante em relação à instituição e sua formação.

No que se refere ao universitário, Astin (1985) e Pascarella (1985) chamam a atenção para a qualidade do esforço estudantil, isto é, o envolvimento do acadêmico com sua própria aprendizagem e desenvolvimento.

A instituição também desempenha um papel essencial para a integração do estudante à vida universitária, facilitando ou não esse processo. A condução adequada desses seqüentes momentos de adaptação pode desencadear no indivíduo o desenvolvimento de repertório para lidar mais adequadamente com novos desafios (Felner \& Adan, 1990).

Essa preocupação tem levado diferentes instituições de ensino superior, principalmente estrangeiras, a desenvolverem programas e/ou criarem serviços para integração do estudante à vida acadêmica. Evidencia-se, assim, a importância de se obter medidas objetivas da integração acadêmica que considere todas as suas dimensões.

$\mathrm{Na}$ revisão para esse trabalho foi localizado o trabalho nacional de Caio (1996) que estudou a adaptação da escala de Baker e Siryk (1989) Student Adaptation to College Questionaire (SACQ) à realidade brasileira. Os resultados mostraram boa qualidade do instrumento mas, como a própria autora refere, necessidade de outras investigações que possam solidificar a utilização da versão brasileira da SACQ quanto à validade, precisão e padronização nacional. $\mathrm{O}$ uso do próprio instrumento de Baker e Siryk visando à continuidade da análise de uma versão nacional, não se mostrou eficaz em razão do fato que a autorização para o uso da escala traria um custo muito alto e seria restrita à realização da pesquisa, sem possibilidade de uso posterior do instrumento. Dessa forma, a opção tomada foi para o investimento na criação de uma escala especialmente destinada à nossa população de universitários, propósito deste trabalho.

\section{Método}

\section{Participantes}

A amostra para esta pesquisa foi composta por 633 universitários provenientes de dois períodos de formação (primeira $[\mathrm{n}=376]$ e penúltima ou última $[\mathrm{n}=257]$ séries) do período noturno de sete cursos agrupados nas áreas de ciências humanas, exatas e biológicas. Participaram 88 estudantes do curso de Psicologia, 68 de Letras, 72 de Análise de Sistemas, 82 de Mecatrônica, 44 de Engenharia da Computação, 150 de Farmácia e 129 de Odontologia, perfazendo um total de 156 acadêmicos da área de Humanas, 198 da área de Exatas e 279 da área de Biológicas.

\section{Materiais e Procedimento}

A Escala de Integração ao Ensino Superior (EIES) foi criada com base nas categorias definidoras das subescalas do SACQ (Student Adaptation to College Questionaire; Baker e Siryk, 1989), que são: ajustamento acadêmico (motivação, investimento, rendimento e ambiente acadêmico), ajustamento relacional-social (geral, relacionamentos e ambiente social), ajustamento pessoal - emocional (psicológico e físico) e aderência (geral e essa Universidade). Os passos seguidos para a construção da escala foram: (a) estudo das dimensões de conteúdo das subescalas do SACQ para a definição da matriz de conteúdo; (b) criação, pelos autores, de oito itens para cada subcategoria a partir das definições e da análise da literatura sobre adaptação acadêmica (Almeida, 1998; Diniz \& Almeida, 1997; Baker \& Siryk, 1989), modelos teóricos sobre o impacto da Universidade (Tinto, 1975, 1987; Pascarela, 1985; Cabrera, Nora \& Castañeda, 1993) e análise de instrumentos nacionais disponíveis sobre o tema (Azzi, Mercuri \& Moran, 1996; Caio, 1996); (c) discussão para o julgamento e a seleção de itens com base nos critérios de clareza, precisão de linguagem, simplicidade e adequabilidade da classificação em relação aos conceitos; (d) organização aleatória dos itens em afirmativas descritivas de 
experiências ligadas a vida universitária para avaliação de concordância por meio de uma escala Likert de 1 (discordo totalmente) a 7 (concordo totalmente); (e) revisão da redação dos itens da escala por um especialista em língua portuguesa, (f) estudo piloto com 12 universitários para avaliação da compreensão das instruções e dos itens, e (g) revisão, redação e editoração final. A escala final foi composta por 46 itens.

A versão final foi aplicada de forma coletiva nas salas de aula dos participantes, após a permissão dos diretores dos cursos envolvidos, agendamento com os professores e a autorização dos próprios estudantes mediante orientação quanto aos objetivos da pesquisa, uso a ser dado às informações obtidas e garantia de sigilo. A fase de coleta de dados ocorreu durante o período de agosto a novembro de 1998.

\section{Resultados}

O propósito da análise dos dados foi investigar a qualidade psicométrica da escala. Efetuaram-se três procedimentos: análise fatorial exploratória, análise de consistência interna e análise fatorial confirmatória dos fatores de segunda ordem.

A análise fatorial exploratória foi feita partindo-se da matriz de correlações entre os itens. Empregou-se o método dos componentes principais e rotação varimax.
Antes de efetuar a análise inverteu-se a pontuação de 18 itens cuja concordância (valor 7 na escala) indicava falta de integração. Essa inversão fez com que as pontuações de todos os itens estivessem no mesmo sentido, ou seja, partindo de 1, indicando falta de integração, a 7 indicando integração muito alta.

A análise fatorial extraiu 13 fatores com eigenvalue maiores do que um. Pôde-se observar que existiram itens com cargas fatoriais maiores do que $0,30 \mathrm{em}$ mais de um fator. Quando isso ocorreu optou-se por considerar o item como pertencente ao fator em que ele apresentava maior carga. Por meio da análise do conteúdo dos itens com altas cargas fatoriais em cada um dos fatores definiram-se seus significados. O Fator 13 continha um único item com carga elevada, por isso foi eliminado. Restaram 12 fatores que compuseram as subescalas do instrumento que passou a ser constituído, portanto, por 45 itens.

Calcularam-se, para cada aluno, 12 escores nas subescalas (escores fatoriais) obtendo-se a média de pontos nos itens que as constituíam. Na Tabela 1 apresentam-se as descrições das subescalas elaborada a partir da análise do conteúdo dos itens e as estatísticas descritivas (valores mínimos e máximos observados, média e desvio padrão). Apresenta-se também o coeficiente de consistência interna (Alfa de Cronbach).

Tabela 1. Estatísticas Descritivas dos Escores Fatoriais e Precisão por Consistência Interna (Alfa de Cronbach) da Escala de Integração ao Ensino Superior.

\begin{tabular}{llrlrrrrr}
\hline Fator & \multicolumn{1}{c}{ Descrição do fator } & Sigla & Min & Max & M & DP & Alfa & $\begin{array}{c}\text { No de } \\
\text { itens }\end{array}$ \\
\hline 1 & Relacionamento com colegas & COL & 1,00 & 7,00 & 5,56 & 1,04 & 0,695 & 6 \\
2 & Ambiente universitário & AMB & 1,50 & 7,00 & 4,82 & 1,11 & 0,736 & 6 \\
3 & Investimento acadêmico & INV & 1,00 & 7,00 & 5,24 & 1,08 & 0,650 & 4 \\
4 & Participação em eventos & EVE & 1,00 & 7,00 & 3,31 & 1,57 & 0,677 & 3 \\
5 & Enfrentamento. & ENF & 1,00 & 7,00 & 4,02 & 1,29 & 0,592 & 4 \\
6 & Aderência à instituição: compro- & ADC & 1,50 & 7,00 & 6,16 & 0,95 & 0,584 & 4 \\
& misso com o curso & & & & & & & \\
7 & Condições físicas & FIS & 1,00 & 7,00 & 3,23 & 1,63 & 0,610 & 3 \\
8 & Estado de humor & HUM & 1,00 & 7,00 & 5,02 & 1,36 & 0,577 & 4 \\
9 & Relacionamento com os & PRO & 1,00 & 7,00 & 4,65 & 1,28 & 0,612 & 3 \\
& professores & & & & & & & \\
10 & Aderência à instituição: compro- & ADG & 1,00 & 7,00 & 6,03 & 1,21 & 0,423 & 3 \\
& misso com a graduação & & & & & & & \\
11 & Apoio familiar & FAM & 1,00 & 7,00 & 5,80 & 1,29 & 0,368 & 3 \\
12 & Satisfação com o curso & SAT & 1,00 & 7,00 & 4,78 & 1,47 & 0,429 & 2 \\
& Escore Total & EIES & 2,62 & 6,64 & 4,96 & 0,67 & 0,864 & 45 \\
\hline
\end{tabular}

O coeficiente de consistência interna para o escore total foi alto $(0,864)$ indicando que os itens se agruparam coerentemente avaliando o mesmo construto, a integração ao ensino superior. Os coeficientes para as subescalas foram mais baixos já que, obviamente, elas contém um número menor 
de itens.

As subescalas com maior concordância foram: ADG e ADC e com menor concordância: EVE, ENF e FIS. $\mathrm{Na}$ última linha da Tabela 1 apresentam-se as estatísticas para o escore total. Observa-se que a média de concordância foi alta $(4,96)$. A distribuição do escore total, conforme pode ser visto na Figura 1, assemelhouse muito à distribuição normal apresentando apenas uma pequena assimetria negativa $(-0,38)$ como efeito da tendência à maior concordância com os itens da escala. Esse fato possivelmente ocorreu por causa do maior número de itens com sentido positivo (27 de 45).

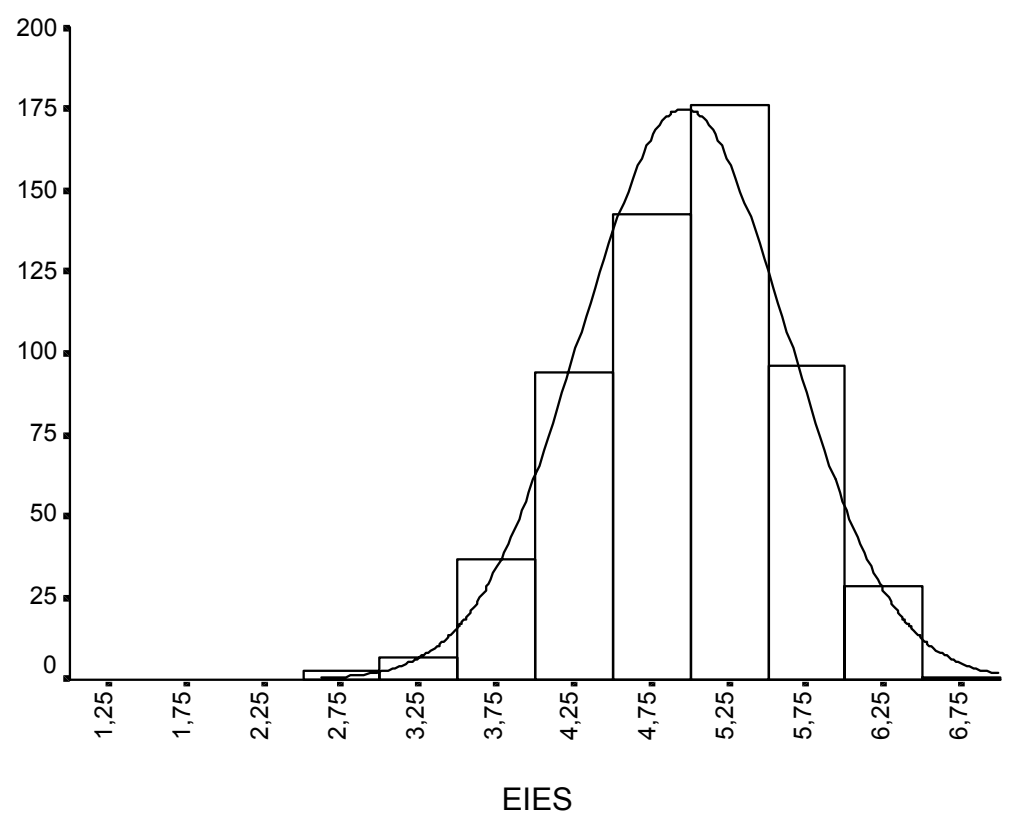

Figura 1. Distribuição dos escores médios da Escala de Integração ao Ensino Universitário.

$\mathrm{Na}$ Tabela 2 apresenta-se a matriz de correlação entre as subescalas. Como pode ser observado os escores fatoriais não foram completamente independentes em parte porque existiram itens com carga alta em mais de um fator.

Tabela 2. Matriz de Correlação entre as Subescalas (escores fatoriais) da EIES.

\begin{tabular}{|c|c|c|c|c|c|c|c|c|c|c|c|c|}
\hline$\overline{\text { Fatores }}$ & 1 & 2 & 3 & 4 & 5 & 6 & 7 & 8 & 9 & 10 & 11 & 12 \\
\hline 1. COL & 1,000 & & & & & & & & & & & \\
\hline 2. AMB & 0,362 & 1,000 & & & & & & & & & & \\
\hline 3. INV & 0,247 & 0,361 & 1,000 & & & & & & & & & \\
\hline 4. ENF & 0,087 & 0,063 & 0,158 & 1,000 & & & & & & & & \\
\hline 5. EVE & 0,269 & 0,271 & 0,388 & 0,107 & 1,000 & & & & & & & \\
\hline 6. ADC & 0,321 & 0,420 & 0,301 & 0,164 & 0,129 & 1,000 & & & & & & \\
\hline 7. FIS & 0,092 & 0,169 & 0,165 & 0,433 & 0,121 & 0,101 & 1,000 & & & & & \\
\hline 8. PRO & 0,421 & 0,353 & 0,271 & 0,176 & 0,235 & 0,226 & 0,005 & 1,000 & & & & \\
\hline 9. HUM & 0,339 & 0,256 & 0,235 & 0,580 & 0,153 & 0,319 & 0,336 & 0,246 & 1,000 & & & \\
\hline 10. ADG & 0,185 & 0,225 & 0,201 & 0,291 & 0,052 & 0,402 & 0,083 & 0,156 & 0,326 & 1,000 & & \\
\hline 11. SAT & 0,289 & 0,331 & 0,111 & 0,228 & 0,015 & 0,286 & 0,109 & 0,205 & 0,308 & 0,279 & 1,000 & \\
\hline 12. FAM & 0,152 & 0,097 & 0,179 & 0,262 & 0,096 & 0,224 & 0,111 & 0,071 & 0,285 & 0,285 & 0,185 & 1,000 \\
\hline
\end{tabular}

A investigação das relações entre as subescalas foi feita pela análise fatorial confirmatória (Long, 1983). Testaram-se dois modelos: (a) o primeiro hipotetizando a exis-tência de um fator geral correspondendo a uma medida ampla da integração ao ensino superior, e (b) o segundo hipotetizando a existência de dois fatores separando os aspectos externos do ambiente acadêmicosocial e internos associados às características pessoais dos 
alunos.

Tabela 3. Índices de Ajuste de dois Modelos de Estrutura Fatorial das Subescalas da Escala de Integração ao Ensino Superior.

\begin{tabular}{llllll}
\hline Modelos & $\chi^{2}$ & $g l$ & $R M S$ & $N F I$ & NPFI \\
\cline { 2 - 6 } Um fator & $560,65^{* * *}$ & 54 & 0,09 & 0,64 & 0,52 \\
Dois fatores & $353,58^{* *}$ & 53 & 0,07 & 0,77 & 0,62 \\
\hline$* * \mathrm{p}<0,01$ & & & & &
\end{tabular}

$\mathrm{Na}$ Tabela 3 apresentam-se os índices de ajuste correspondentes aos dois modelos. Como pode ser observado os valores do Qui Quadrado são, em ambos os casos, significativos indicando que os dois modelos falharam em reproduzir adequadamente a estrutura de correlações entre as subescalas. Entretando como argumenta Mulaik et al. (1989) é útil verificar quão próximo um modelo reproduziu as correlações mesmo diante de valores significativos do Qui Quadrado. Os valores do Índice de Ajuste Normalizado (NFI, Normed Fit Index, Mulaik et al.) indicam que o modelo com dois fatores propiciou uma representação mais próxima do que o modelo com um fator, embora, ele não tenha atingido níveis de ajuste satisfatórios.

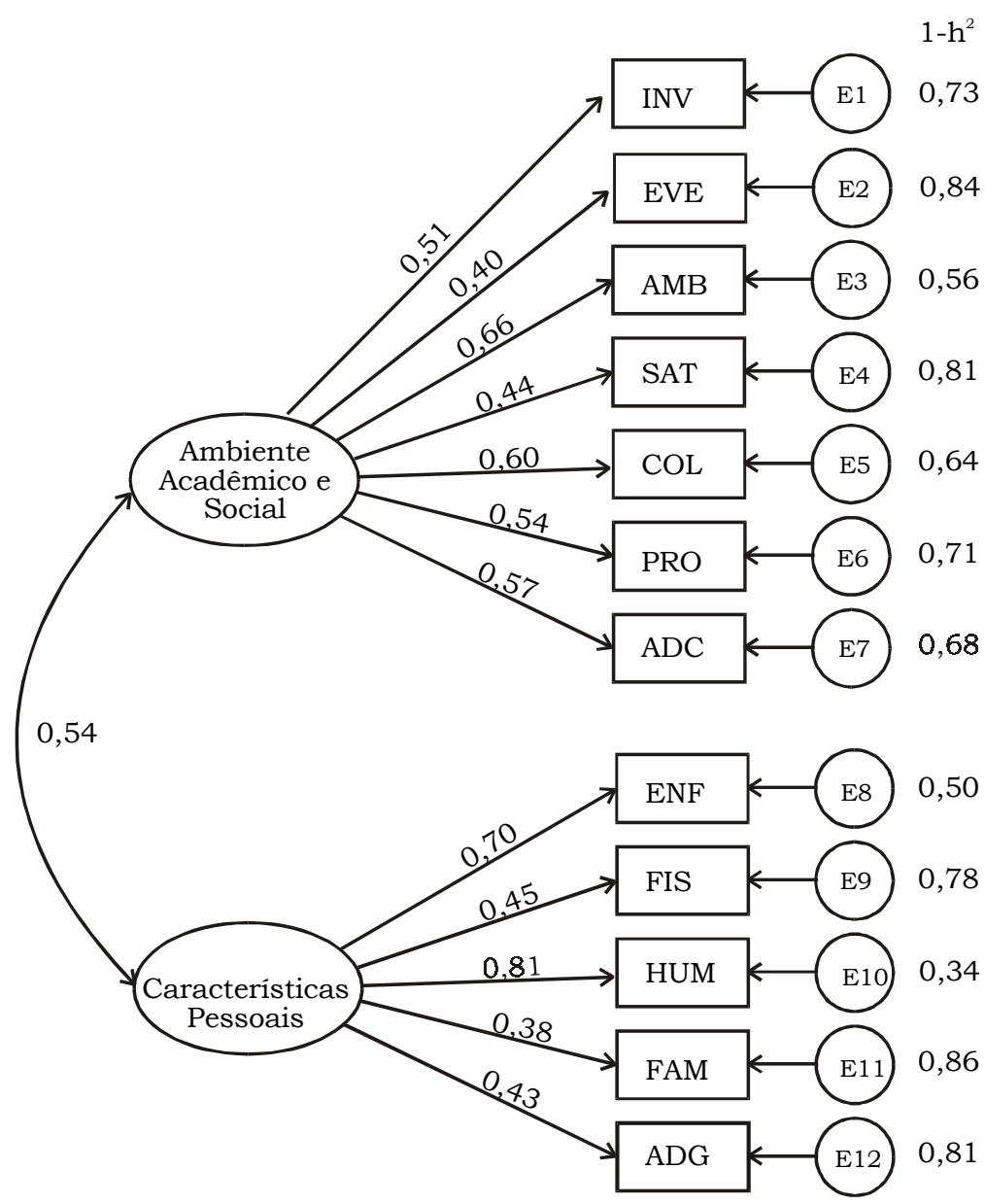

Figura 2. Diagrama de Relações com os Coeficientes Estimados pela Análise Fatorial Confirmatória entre as Subescalas da Escala de Integração ao Ensino Superior.

$\mathrm{Na}$ Figura 2 foi apresentada a representação diagramática do modelo com dois fatores, bem como os parâmetros estimados pela análise fatorial confirmatória. Pode-se observar que as subescalas mais importantes na definição do fator ambiente acadêmico e social são: AMB, COL e ADC. Já para o fator 
características pessoais são: ENF e HUM. As demais subescalas apresentam correlações moderadas ou baixas com seus respectivos fatores. Isso indica que embora relacionadas com a integração acadêmica, grande parte da sua variabilidade não se limita a esse constructo, como mostram as especificidades obtidas $\left(1-\mathrm{h}^{2}\right)$.

\section{Discussão}

Este trabalho apresenta o desenvolvimento de um instrumento de avaliação da integração ao ensino superior e investiga suas propriedades psicométricas.

Este trabalho pauta-se na grande valia que um instrumento como esse pode ter como ferramenta para a identificação da integração à vida universitária, seja para fins de pesquisa ou para intervenções dirigidas ao estudante. Ela poderá se tornar de grande utilidade aos profissionais ligados a serviços de orientação ao universitário, por exemplo.

A escala é de fácil aplicação e compreensão, podendo ser aplicada coletivamente em um tempo de 10 a 20 minutos. Seu formato permite inclusive, a informatização tornando sua aplicação e correção ainda mais simples.

O estudo efetuado demonstrou que a escala, de modo geral, possui qualidade psicométrica satisfatória. Quanto à consistência interna pode-se fazer uma comparação com o trabalho de Baker e Siryk (1989). Esse instrumento, composto por 67 itens e com escala Likert de nove pontos, apresenta consistência interna entre 0,92 a 0,95 . A escala apresentada aqui é composta por um número menor de itens e de pontos na escala Likert, fatores que tendem a reduzir a consistência interna. Mesmo assim a precisão permaneceu alta atingindo o coeficiente de 0,86 .

Esse estudo aponta a existência de dois grandes fatores relacionados à integração ao ensino superior: (a) o primeiro associado sobretudo aos aspectos externos relacionados ao ambiente universitário, de satisfação com o curso e, portanto, aderência ao mesmo, e (b) o segundo, sobretudo, aos aspectos internos do indivíduo, de capacidade de enfrentamento, reações físicas psicossomáticas e estado de humor. Esses fatores podem, ainda, ser subdivididos em 12 facetas para uma compreensão mais detalhada da vivência de um determinado estudante na universidade. Entretanto a interpretação das facetas deve ser feita com cautela pela baixa consistência interna já que os escores das subescalas são baseados em uma amostra reduzida de respostas (isto é, baixo número de itens).

Ainda são necessários estudos de consistência temporal para verificar em que medida a integração ao ensino superior se altera ao longo do tempo e que condições conduzem a essa alteração. Outro aspecto refere-se a validade de critério. É necessário demonstrar como os escores obtidos pela escala se relacionam a outras variáveis associadas a integração como, por exemplo, o desempenho acadêmico e a evasão.

Em suma, o instrumento mostrou possuir boa qualidade motivando o prosseguimento de estudos que possam aprofundar as informações a seu respeito. Em especial, quanto ao propósito deste trabalho, a escala se mostrou útil ao projeto mais amplo de caracterização psicológica do estudante universitário, pois apresentou propriedades psicométricas satisfatórias, justificando seu uso como medida dependente da integração ao ensino superior.

\section{Referências}

Almeida, L. S. (1998). Adaptação, rendimento e desenvolvimento dos estudantes do ensino superior: estudo junto dos alunos do $1^{\circ}$ ano da Universidade do Minho - relatório técnico da investigação. Centro de Estudos em Educação e Psicologia. Instituto de Educação e Psicologia. Universidade do Minho. Braga, abril.

Arruda, P. V. de A.; Millan, L. R.; Rossi, E.; Marco, O. L. N. (1994). Introdução. Documentos do CEDEM, (5): 1-4. São Paulo, CEDEM/FCM/USP.

Astin, A. W. (1985). Achieving Educational Excellence: A Critical Assessment of Priorities and Practices in Higher Education. San Francisco: Jossey-Bass.

Azzi, R. G.; Mercuri, E.; Moran, R. C. (1996). Fatores que interferem na decisão de desistência de curso no primeiro ano de graduação. Campinas. 8p. (Mimeo).

Baker, R. W. \& Siryk, B. S. (1989). SACQ Student adaptation to college questionaire: Manual. Los Angeles (CA): Western Psychological Services, WPS.

Bean, J. P. (1980). Dropouts and turnover: the synthesis and test of a causal modelo of student attrition. Research in Higher Education, 12 (2):155-187.

Bezzon, L. A. C. (1995). Análise do perfil sócio-econômico cultural dos ingressantes na Unicamp (1987-1994): democratização on eletização? São Paulo, Dissertação (mestrado). Instituto de Filosofia e Ciências Humanas da Unicamp. 248p.

Brower, A. M. (1992). The "second half" of student integration: the effects of life task predominance on student persistence. Journal of Higher Education, 63 (4): 441 462.

Cabrera, A. F.; Castañeda, M. B.; Nora, A.; Hengstler, D. (1992). The convergence between two theories of college persistence. Journal of Higher Education, 63 (2): 143-164.

Cabrera, A. F.; Nora, A.; Castañeda, M. B. (1993). College persistence. Journal os Higher Education, 64 (2): 123-139.

Caio, V. M. (1996). Integração do estudante à Universidade: um estudo exploratório. Dissertação de Mestrado. Campinas, Pontifícia Universidade Católica de Campinas, São Paulo. xiv 100p.

Calejon, L. M. C. (1996). Manejo de crises e dificuldades adaptativas em universitários. Tese de Doutorado. Instituto de 
Psicologia da Universidade de São Paulo, São Paulo.

Carelli, M. J. G. (1996). Perfil sócio-cultural e condições de estudo de alunos da USF. Mestado em Educação. F.F.C.L., USF.

Christie, N. G. E Dinham, S. M. (1991). Institutional and external influences on social integration in the freshman year. Journal of Higher Education, 62 (4): 412-436.

Diniz, A. M. e Almeida, L. S. (1997). Construção de uma escala de qualidade da integração no ensino superior (EQIES). Psicologia: teoria, investigação e prática, 2: 85-96.

Felner, R. D. e Adan, A. M. (1990). The school transitional enviroment project: an ecological intervention and evaluation. In: Price, R. H.; Cowen, E.; Lorion, R.P.; Ramos, M. Fourteen onces of primary prevention. Washington, American Psychology Association. p. 111-122.

Frost, S. (1993). Strategies to help freshmen succeed. Planning for Higher Education, 21(4):21-26.

Gerder, H. e Mallinckrodt, B. (1994). Emotional, social and academic adjustment of college students: a longitudinal study of retention. Journal of Counseling and Development, 72: 281-288.

Gunter, I. de A. E Gunter, H. (1986). Desenvolvimento adulto entre estudantes brasileiros nos EUA: busca de um modelo. Estudos de Psicologia, Campinas, 3(1-2):84-105.

Larose, S. e Roy, R. (1991). The role of prior academic performance and non-academic attributes in the prediction of the sucess og high-risk college students. Journal of College Student Development, 32: 171-177.

Long, J. S. (1983). Confirmatory factor analysis. Sage University Paper series on Quantitative Application in the Social Sciences, 07-033. Beverly Hills and London: Sage Pubns.

Mercuri, E. N. G. S. (1992). Condições espaciais, materiais e pessoais para o estudo, segundo depoimentos de alunos e professores de graduação da Unicamp. Tese de Doutorado. Universidade Estadual de Campinas. Faculdade de Educação. Campinas, SP.

Mercuri, E. N. G. S.; Moran, R. C.; Azzi, R. G. (1995). Estudo da evasão de curso no primeiro ano da graduação de uma Universidade pública estadual. NUPES. São Paulo, NUPES/USP. 13p. (Documento de Trabalho 05/95)
Millan, L. R. E Barbedo, M. F. (1994). Assistência psicológica ao aluno de Medicina: o início de uma experiência. Documentos do CEDEM, (5): 9-18. São Paulo, CEDEM/FCM/USP.

Mulaik, S. A.; James, L. R.; Alstine, J. V.; Bennett, N.; Lind, S. \& Stilwell, C. D. (1989). Evaluation of goodness of fit indices for structural equation models. Psychological Bulletin, 105, 3, 430-445.

Pacheco, E. M. de C. (1996). Indicadores de risco de sucesso acadêmico segundo universitários. São Paulo, 144p. Dissertação (Mestrado). Instituto de Psicologia da Pontifícia Universidade Católica de Campinas.

Pascarella, E. T. (1985). Students' affective development within the college environment. Journal of Higher Education, 56: 649-663.

Primi, R.; Vendramini, C. M.; Santos, A. A. A. \& Figueiredo, N. (1999). Impacto de variáveis sócio econômicas no desempenho de candidatos ao ensino superior. Em A. P. Soares; S. Araújo; S. Caires (Orgs.). Avaliação Psicológica: Formas e Contextos Volume VI. (pp. 195-202). Braga, Portugal: APPORT, Associação dos Psicólogos Portugueses.

Santos, A. A. A.; Primi, R.; Vendramini, C. M.; Taxa, F.; Lukjanenko, M. F., Muller, F., Sampaio, I.; Andraus Jr., S.; Kuse, F. K. \& Bueno, C. H. (2000). Habilidades básicas de ingressantes universitários. Avaliação: Revista da Rede de Avaliação Institucional da Educação Superior, 2(16): 33-45.

Terenzini, P. T.; Rendon, L. I.; Upcraft, M. L.; Millar, S. B.; Allinson, K. W.; Gregg, P. L.; Jalomo, R. (1994). The transiton to college: diverse students, diverse stories. Research in Higher Education, 35 (1): 57-73.

Tinto, V. (1975). Dropout from higher education: a theorical synthesis of recent research. Educational Research, 45: 89125.

Tinto, V. (1987). Leaving college: rethinking the causes and cures of student attrition. Chicago, IL: University os Chicago Press.

Recebido em 15/05/2000

Revisado em 14/05/2001

Aceito em 21/06/2001

Sobre os autores:

Soely A. J. Polydoro é Psicóloga, Doutora em Psicologia Educacional pela Universidade Estadual de Campinas. Professora e Coordenadora do Curso de Graduação em Psicologia da Universidade São Francisco.

Ricardo Primi é Psicólogo, Doutor em Psicologia Escolar e do Desenvolvimento Humano pela Universidade de São Paulo com parte desenvolvida na Yale University (EUA). Professor do Curso de Graduação e do Mestrado em Psicologia da Universidade São Francisco.

Maria de Nazaré da F. Serpa é Docente do Centro de Ciências Humanas e Sociais e psicóloga do Serviço de Orientação ao Estudante da Universidade São Francisco.

Margarida M. Hoeppner Zaroni é Docente do Centro de Ciências Humanas e Sociais da Universidade São Francisco.

Kelly Cristina Pereira Pombal é Acadêmica do curso de Psicologia do Centro de Ciências Humanas e Sociais da Universidade São Francisco e Bolsista de Iniciação Científica (PROBAIC/USF). 